\title{
On Parsing and Compiling Arithmetic Expressions on Vector Computers
}

\author{
CHARLES N. FISCHER \\ University of Wisconsin-Madison
}

The problem of parsing and compiling arithmetic expressions on vector computers is considered. Methods are developed which allow encodings of one or more arithmetic expressions to be transformed directly into encodings of their corresponding derivation trees. The algorithm which performs this transformation is compact, efficient, and able to make extensive use of concurrent vector operations. Routines which concurrently transverse encoded derivation trees in a top-down or bottom-up manner are presented. These routines can be used to structure efficient, compact, and highly concurrent algorithms which complete the process of compiling arithmetic expressions.

Key Words and Phrases: vector parsing, vector compiling, arithmetic infix expressions, vector computers

CR Categories: $4.12,5.23,5.25$

\section{INTRODUCTION}

With the advent of vector computers such as the CDC Star-100 [20], TI ASC [1], and Cray-1 [7], the design and analysis of algorithms which exploit the concurrency possible in vector machines has become of great interest. Rather surprisingly, however, the problem of designing compilers to run on vector computers has received relatively little attention $([3,8,9,12,14,15,21])$.

In this paper we consider the problem of parsing and compiling a very general class of arithmetic infix expressions on vector machines. Such expressions are of interest in that they are an integral part of virtually all programming languages; in fact, in some languages (such as APL) they represent the only nontrivial structure present. Thus they represent a natural first step in the investigation of how to implement compilers on vector computers. We suggest that all the arithmetic expressions occurring in a program segment, or perhaps an entire program, could be parsed (or compiled) concurrently in an efficient manner via suitable vector operations.

A model is considered in which scalars (denoted by lowercase identifiers) and vectors (denoted by uppercase identifiers) are the available primitive data objects. Componentwise operations between two vectors or between a vector and a scalar

Permission to copy without fee all or part of this material is granted provided that the copies are not made or distributed for direct commercial advantage, the ACM copyright notice and the title of the publication and its date appear, and notice is given that copying is by permission of the Association for Computing Machinery. To copy otherwise, or to republish, requires a fee and/or specific permission.

Author's address: Department of Computer Sciences, University of Wisconsin-Madison, 1210 W. Dayton St., Madison, WI 53706.

(C) 1980 ACM 0164-0925/80/0400-0203 $\$ 00.75$ 
are assumed to proceed with a significant degree of concurrency (typically via pipeline techniques [17]). Such operations include componentwise comparison and arithmetic operations as well as vector assignment, compression, and indexing. ${ }^{1}$ Further, the model assumes that the amount of concurrency available is bounded, that is, that vector operations require a time proportional to the size of the vectors involved to proceed. We believe this model of vector computers represents a very useful and general abstraction of the properties of such machines.

Rather informally, an algorithm will be considered to be highly concurrent if it makes extensive use of the concurrent vector operations assumed available. Naturally, the actual degree of concurrency realized on a particular machine will depend heavily upon machine-specific details. Thus the algorithms and techniques developed here will place special emphasis on the use of vectors and vector operations in parsing and compilation as the primary means of exploiting the concurrency available in vector machines.

First, arithmetic infix grammars (AIGs) are introduced as a means of formally specifying the exact class of arithmetic expressions to be processed. Next, it is seen that any number of arithmetic expressions can be parsed efficiently and with a high degree of concurrency. In particular, vector encodings of such expressions can be transformed directly into encodings of their corresponding derivation trees. This avoids the overhead, inherent in serial parsing techniques, of repeatedly finding and replacing single occurrences of various productions.

Finally, it is seen that encoded derivation trees can be traversed in either a top-down or a bottom-up manner with a high degree of concurrency. This allows typical semantic processing of expressions (type checking, type conversions, etc.) to be performed effectively. Further, during traversals of encoded derivation trees, code generation questions (allocation of temporary locations, ordering of generated code, etc.) are also discussed. In short, almost all the operations required to translate arithmetic expressions are seen to be efficiently realizable via vector operations.

\section{ARITHMETIC INFIX GRAMMARS}

The structure of an arithmetic infix expression is determined by the properties of its operators. Usually the set of operators available is partitioned into a number of classes. Each operator class is distinguished by its kind (binary or unary), its priority (often called precedence), and its associativity (for binary operators either right or left). Arithmetic infix expressions also typically assume the ability to index arrays and to invoke functions.

Indexed arrays typically are of the form $A[B, C, \ldots]$, while function invocations are commonly of the form $f(x, y, \ldots)$. To put these items into a standard infix form, we assume that the scanner (or a prepass) does the following transformations:

(1) change all occurrences of "[" to "index (";

\footnotetext{
${ }^{1}$ The Cray-1 does not allow vector compression or vectors of indices. However, these omissions are considered a serious design weakness [4].
} 
(2) change all ]'s to ' 's; ${ }^{2}$

(3) change all occurrences of "(" which follow an ID, ")" or "]" to "arg (";

(4) change all occurrences of "," to ") apply (".

Thus $A[B, C, \ldots]$ becomes $A$ index $(B)$ apply $(C)$ apply (..), while $f(x, y, \ldots)$ becomes $f \arg (x)$ apply $(y)$ apply (...).

Note that each of the above transformations is determined by local context only. This allows them to be readily and efficiently realized using vector operations. index, arg, and apply are assumed to be predefined (as detailed below). index applies a subscript to an array, reducing its dimensionality by 1 . arg binds an actual parameter to a formal parameter. When all formal parameters have been bound, a call of the function may be made. apply may be used either as index or arg (i.e., it is overloaded). Its usage is determined during semantic processing (see Section 6) by the type (array or function) of its left operand.

Observe that the case in which parentheses are used to delimit both array indices and function parameters (as occurs in Fortran) is readily handled. Transformations (1) and (2) are eliminated while (3) replaces appropriate ('s with "apply (". If functions of no arguments are allowed, two possibilities occur. If a null parameter list is required (e.g., $f()$ as found in the programming language BCPL [18]), then a transformation of $f()$ to $f$ arg NULL_LIST can be done. Otherwise, such functions are syntactically identical to ordinary variables and must be invoked as a type conversion during semantic processing (see Section 6).

An arithmetic infix language can be completely characterized by $n+1$ pairwise disjoint operator classes $\mathrm{OP}_{0}, \ldots, \mathrm{OP}_{n}$. Operators in $\mathrm{OP}_{i+1}$ will have a higher priority than those in $\mathrm{OP}_{i}, \mathrm{OP}_{i-1}$, etc. The highest priority operators are applied first, then the next highest, etc. Each operator class, $\mathrm{OP}_{i}$, has a kind, KIND[i], which is either BINARY or UNARY. Each class also has an associativity, ASSOC $[i]$. A -1 denotes left associativity; a 1 denotes right associativity. If a class $\mathrm{OP}_{i}$ has $\mathrm{KIND}[i]=\mathrm{UNARY}$, then ASSOC $[i]$ must be 1 (all unary operators are right associative). We predefine the class $\mathrm{OP}_{0} \equiv\{\#\} . \#$ is a binary, left associative null operator used as an endmarker and as a separation between adjacent arithmetic expressions. We also predefine $\mathrm{OP}_{n} \equiv$ index, arg, apply\}. These operators are binary and left associative.

A given operator class is not allowed to contain both a binary operator $\theta$ and a unary operator $\gamma$ because of the ambiguity in parsing, for example, $\gamma a \theta b$. In like manner, a class may not contain both a left associative binary operator $\theta$ and a right associative binary operator $\gamma$ because of the ambiguity in parsing $a \theta b \gamma c$. We assume that all operators are distinct and that each can be uniquely associated with some class. It is common practice to use the same symbol to represent both a binary and unary operator (e.g., + or -). This can be dealt with in the scanner (or in a transformation operation) by replacing such symbols with unique operators depending upon whether they are used as unary or binary operators. It is easy to discern such usage as binary operators are always preceded

\footnotetext{
${ }^{2}$ Note that this transformation may have the effect of changing an illegal construct into a legal one (e.g., $A(X]$ into $A(X)$ ). This is a minor point as syntax checks will detect unbalanced parentheses. However, if desired, a left-to-right pass to check for this error can be performed before the transformation.
} 
by a ')', ']', or identifier, while unary operators must be preceded by an operator, '(', or '['.

For example, an arithmetic infix language having the operators,+- , ident (unary +), neg (unary - ), *, /,**, and the usual semantics would be characterized as follows:

$\mathrm{OP}_{0}=\{\#\}, \quad \mathrm{OP}_{1}=\{+,-\}, \quad \mathrm{OP}_{2}=\{$ ident, neg $\}, \quad \mathrm{OP}_{3}=\{*, /\}, \quad \mathrm{OP}_{4}=\{* *\}, \quad \mathrm{OP}_{5}=$ \{index, arg, apply\}

$\mathrm{KIND}[0]=\mathrm{KIND}[1]=\mathrm{KIND}[3]=\mathrm{KIND}[4]=\mathrm{KIND}[5]=\mathrm{BINARY}$

$\mathrm{KIND}[2]=\mathrm{UNARY}$

ASSOC $[0]=\operatorname{ASSOC}[1]=\operatorname{ASSOC}[3]=\operatorname{ASSOC}[5]=-1$

$\operatorname{ASSOC}[2]=\operatorname{ASSOC}[4]=1$

We may now define a context-free grammar [10] called an arithmetic infix grammar which formally specifies the arithmetic infix language characterized by $\mathrm{OP}_{0}, \ldots, \mathrm{OP}_{n}, \operatorname{ASSOC}[0], \ldots, \operatorname{ASSOC}[n]$, and $\mathrm{KIND}[0], \ldots, \mathrm{KIND}[n]$ as $G=$ $\left(V, V_{T}, P, S\right)$.

$$
\begin{aligned}
V_{T} & =\{\mathrm{ID},(,)\} \cup \mathrm{OP}_{0} \cup \ldots \cup \mathrm{OP}_{n} \\
V & =V_{T} \cup\left\{S, N_{0}, \cdots, N_{n+1}\right\} .
\end{aligned}
$$

$P$ is the union of the following sets of productions:

(1) $\left\{S \rightarrow \# N_{0} \#\right\}$;

(2) $\left\{N_{i} \rightarrow N_{i+1} \mid 0 \leq i \leq n\right\}$;

(3) $\left\{N_{n+1} \rightarrow\right.$ ID, $\left.N_{n+1} \rightarrow\left(N_{1}\right)\right\}$;

(4) for $0 \leq i \leq n$ :

$$
\begin{aligned}
& \text { if KIND }[i]=\text { UNARY then }\left\{N_{i} \rightarrow \theta N_{i} \mid \theta \in \mathrm{OP}_{i}\right\} \\
& \text { else if ASSOC[i]=-1 } \\
& \text { then }\left\{N_{i} \rightarrow N_{i} \theta N_{i+1} \mid \theta \in \mathrm{OP}_{i}\right\} \\
& \text { else }\left\{N_{i} \rightarrow N_{i+1} \theta N_{i} \mid \theta \in \mathrm{OP}_{i}\right\}
\end{aligned}
$$

In the following sections we will find AIGs useful in establishing the correctness of various algorithms. We may also readily observe that all AIGs are unambiguous for we can easily establish that they are LR(1) [2].

\section{TESTING FOR WELL-FORMED ARITHMETIC INFIX EXPRESSIONS}

In general, a parser serves two functions. It verifies that an input string is, in fact, in $L(G)$ (that is, derivable from $S$ ) and, given that it is, it produces a parse of the string.

If $G$ is an AIG then, as we shall see, it is simple to test whether an input string is in $L(G)$. This follows from the fact that $L(G)$ is rather uniform in structure. The following definitions will allow us to formulate an easy test of whether or not a string is in $L(G)$. Let $U \subseteq V_{T}$ for some AIG. Define Follow $(U)=\left\{b \in V_{T} \mid\right.$ $S \rightarrow^{+} \ldots a b \cdots$ and $\left.a \in U\right\}$. Follow $(U)$ is the set of terminal symbols that may legitimately follow some symbol in $U$. Let $\mathrm{UN}=\left\{\theta \in \mathrm{OP}_{i} \mid 0 \leq i \leq n\right.$ and KIND[i] $=\mathrm{UNARY}\}$ and $\mathrm{BIN}=\left\{\theta \in \mathrm{OP}_{i} \mid 0 \leq i \leq n\right.$ and $\left.\mathrm{KIND}[i]=\mathrm{BINARY}\right\}$. It can be easily verified that the Follow sets listed below are correct.

Set: UN BIN $\{(\}\{)\}$ (ID\}

Follow: $F 1 \quad F 1 \quad F 1 \quad F 2 \quad F 2$

where $F 1 \equiv \mathrm{UN} \cup\{(, \mathrm{ID}\}$ and $F 2 \equiv \mathrm{BIN} \cup\{)\}$. 
Next, let $a \in V_{T}$ and $x \in V_{T}^{+}$. Define $\operatorname{COUNT}(x, a)$ as the number of occurrences of $a$ in $x$. For example, COUNT $(a a b a a, a)=4$ and $\operatorname{COUNT}(a a b a a, c)=0$. For an operator $\theta$ let CLASS[ $\theta]=j$ if and only if $\theta \in \mathrm{OP}_{j}$. Define CLASS[ID] as $n+1$ and CLASS[(] $=$ CLASS[)] $=n+2$. ASSOC and KIND are extended so that $\operatorname{ASSOC}[n+1]=\operatorname{ASSOC}[n+2]=1, \operatorname{KIND}[n+1]=\operatorname{IDENTIFIER}$ and $\mathrm{KIND}[n+2]=$ OTHER.

Finally, let $x \in V_{T}^{*}$ for some AIG (call it $G$ ). Then $x$ is well formed (with respect to $G$ ) if and only if

(1) $x$ is of the form $\# y \#$ for $y \in V_{T}^{*}$;

(2) $x=x_{1} x_{2} \Rightarrow \operatorname{COUNT}\left(x_{1}\right.$, , (') $^{\prime} \operatorname{COUNT}\left(x_{1},{ }^{\prime}\right)$ ') $\geq 0$ for $x_{1} \in V_{T}^{+}, x_{2} \in V_{T}^{*}$;

(3) $x=x_{3} \# x_{4} \Rightarrow \operatorname{COUNT}\left(x_{3} \#\right.$, '(') $-\operatorname{COUNT}\left(x_{3} \#\right.$, ' ')' $)=0$ for $x_{3}, x_{4} \in V_{T}^{*}$;

(4) $x=\cdots a b \cdots$ for $a, b \in V_{T} \Rightarrow$

(i) $b \in$ Follow $(\{a\})$;

(ii) $b \in \mathrm{UN}$ and $a \in(\mathrm{UN} \cup \mathrm{BIN}) \Rightarrow \operatorname{CLASS}[b] \geq \operatorname{CLASS}[a]$.

Condition (1) requires correct endmarkers, while conditions (2) and (3) require proper parenthesis structure. Condition (4ii) forbids certain illegal operator pairs. The following establishes the importance of well formedness.

LEMma 3.1 Let $G$ be an $A I G$. Then for any $x \in V_{T}^{*}, x \in L(G)$ if and only if $x$ is well formed.

Proof. To prove $x \in L(G) \Rightarrow x$ is well formed, we proceed as follows. Conditions (1), (2), (3), and (4i) are obvious. Further, observe that whenever an operator in class $i$ is generated, it is followed by $N_{j}$ where $i \leq j \leq i+1$. But if $N_{j}$ generates a unary operator as its first (i.e., leftmost) symbol, it must be in a class $\geq j$. Thus condition (4ii) must hold.

To prove $x$ is well formed $\Rightarrow x \in L(G)$, write $x$ as $\# x_{1} \# x_{2} \# \ldots \# x_{m} \#$, where \# does not occur in $x_{1}, \ldots, x_{m}$. Write $x_{1}$ as $w(y) z$, where $y$ contains no '(' or ')'; otherwise, let $y=x_{1}$. Reduce all IDs in $y$ to $N_{n+1}$. Using Follow sets, $y$ must be of the form $\left(\mathrm{UN}_{n}^{*} N_{n+1}\right)\left(\mathrm{BIN}_{n} \mathrm{UN}_{n}^{*} N_{n+1}\right)^{*}$, where for $0 \leq i \leq n, \mathrm{UN}_{i} \equiv \mathrm{UN} \cap$ $\left(\mathrm{OP}_{0} \cup \ldots \cup \mathrm{OP}_{i}\right)$ and $\mathrm{BIN}_{i} \equiv \mathrm{BIN} \cap\left(\mathrm{OP}_{0} \cup \ldots \cup \mathrm{OP}_{i}\right)$. We now show that for any $j, 1 \leq j \leq n+1, y$ can be reduced to the form $\left(\mathrm{UN}_{j-1}^{*} N_{j}\right)\left(\mathrm{BIN}_{j-1} \mathrm{UN}_{j-1}^{*} N_{j}\right)^{*}$. This clearly holds for $j=n+1$ as noted above. Assume this holds for $j>1$ and the following will show $y$ can be reduced to satisfy the form for $j-1$.

If $y$ contains no operators in $\mathrm{OP}_{j-1}$, we merely reduce all $N_{j}$ 's to $N_{j-1}$. Otherwise, consider $\operatorname{KIND}[j-1]$. If it is UNARY, then reduce all $N_{j}$ 's to $N_{j-1}$ and consider each substring of $y$ matched by $\mathrm{UN}_{j-1}^{*} N_{j-1}$. If any operators in $\mathrm{OP}_{j-1}$ are matched, the operator to the immediate left of $N_{j-1}$ must be in $\mathrm{OP}_{j-1}$ (otherwise condition (4ii) would be violated). This operator and $N_{j-1}$ can be reduced to $N_{j-1}$ and this can be repeated until all operators in $\mathrm{OP}_{j-1}$ are reduced. The result clearly satisfies the desired form for $j-1$.

If $\mathrm{KIND}[j-1]=\mathrm{BINARY}$, then consider in turn maximal substrings of $y$ matched by $N_{j}\left(\mathrm{OP}_{j-1} \mathrm{UN}_{j-1}^{*} N_{j}\right)^{+}$. By condition (4ii), $\mathrm{UN}_{j-1}^{*}$ must match $\lambda$ (the empty string). Thus these substrings are of the form $N_{j}\left(\mathrm{OP}_{j-1} N_{j}\right)^{+}$and can clearly be reduced to $N_{j-1}$. When all operators in $\mathrm{OP}_{j-1}$ are reduced, any remaining $N_{j}$ 's can be reduced to $N_{j-1}$ and the desired form is obtained for $j-1$. 
Thus $y$ can be reduced to the form $\left(\mathrm{UN}_{0}^{*} N_{1}\right)\left(B I N_{0} U N_{0}^{*} N_{1}\right)^{*}$. But $y$ contained no \#'s so this reduces to $N_{1} . x_{1}$ has either been reduced to $N_{1}$ or to $w\left(N_{1}\right) z$. In the latter case we reduce this to $w N_{n+1} z$ and iterate the above until $x_{1}$ is reduced to $N_{1}$. In like manner $x_{2}, \ldots, x_{m}$ are reduced to obtain $\# N_{1} \# \ldots \# N_{1} \#$ which can then be reduced to $S$. Thus $S \rightarrow^{+} x$.

We now show that well formedness can be tested in an efficient and highly concurrent manner. Obviously condition (1) is trivial. Let $|V|$ denote the size of vector $V$ and assume ALL_ONES $(B)$ returns 1 (representing true) if and only if bit vector $B$ (composed of only 0's and 1's) contains only 1's. Assume for any symbol $a \in V_{T}$, FOLLOW [ $a$ ] yields $F 1$ or $F 2$ (as defined above) and F_CLASS[ $\left.a\right]$ yields the follow class ( $F 1$ or $F 2$ ) to which $a$ belongs. The condition (4i) is tested on string INPUT by

$$
\begin{aligned}
& \text { ALL_ONES(FOLLOW[INPUT }[1, \ldots, \mid \text { INPUT } \mid-1]] \text { = F_CLASS[INPUT }[2, \ldots, \mid \\
& \text { INPUT |]]) }
\end{aligned}
$$

Condition (4ii) can be tested in a similar manner.

Let $B$ be a bit vector and $V$ an arbitrary vector such that $|B|=|V|$. Define $B$ compress $V$ as a vector composed of those elements of $V$ whose corresponding $B$ elements are 1 . Thus $1,0,1,1$ compress $1,2,3,4,=1,3,4$. Condition (4ii) can then be tested by

1. CKPOS := ((CLASS[INPUTT $[1, \ldots,|\operatorname{INPUT}|-1]] \leq n)$ and (KIND[CLASS[INPUT[ $2, \ldots, \mid$ INPUT $\mid]]]=$ UNARY $))$ compress $(2, \ldots, \mid$ INPUT $\mid)$

2. ALL_ONES(CLASS[CKPOS] $\geq$ CLASS[CKPOS - 1])

Finally, let COUNT_VECT (INPUT, $a$ ) $=$ COUNT(INPUT[1], $a$ ), COUNT((INPUT[1], INPUT[2]), $a), \ldots$, COUNT(INPUT, $a$ )

Then condition (2) can be tested by

ALL_ONES ((COUNT_VECT(INPUT,'(') - COUNT_VECT (INPUT,')')) $\geq 0$ )

Condition (3) can be tested by

ALL_ONES (INPUT $=$ '\#’) compress ((COUNT_VECT (INPUT, ' (') COUNT_VECT(INPUT,')')) =0))

We may use the following algorithm to compute COUNT_VECT.

Algorithm 3.2 (Compute COUNT_VECT $(X, a)$ )

1. $V[1,3, \ldots, 2 *|X|-1]:=X$

$V[2,4, \ldots, 2 *|X|]:=$ MARK. $^{3}$

2. $V:=(V \neq a)$ compress $V$.

3. COUNT_VECT $:=(2,4, \ldots, 2 *|X|)-((V=$ MARK $)$ compress $(1,2, \ldots,|V|))$.

Note that Algorithm 3.2 uses the fact that after removing all $a$ 's from $V$ (in step 2), the $i$ th MARK in $V$ has been shifted left by exactly COUNT((X[1], .., $X[i]), a)$. Finally, observe that the well-formedness tests presented are both

\footnotetext{
${ }^{3}$ Let MARK be some value known not to occur in $X$.

ACM Transactions on Programming Languages and Systems, Vol. 2, No. 2, April 1980.
} 
efficient (clearly linear in the size of INPUT) and highly concurrent (in that vector operations are used extensively).

\section{PARSING ARITHMETIC INFIX EXPRESSIONS}

We are now ready to attack our main objective, that of parsing well-formed arithmetic infix expressions. The order of application of operators in an expression depends on a number of factors:

(1) the depth of parenthesis nesting;

(2) the relative priorities of the operators;

(3) the associativity of the operators;

(4) the positions of the operators and identifiers in the expression.

We shall encode these factors, uniquely, for each operator and identifier in an integer, termed its precedence.

Let INPUT be a well-formed input string. Define INDEX $=$ (CLASS[INPUT] $\leq n+1)$ compress $(1,2, \ldots, \mid$ INPUT $\mid)$ and ID_OPS $=$ INPUT[INDEX]. ID_OPS are the identifiers and operators occurring in INPUT, and INDEX contains their positions in INPUT. We can now encode the four factors noted above as

(1) NEST(ID_OPS) $=$ (COUNT_VECT(INPUT,'(') - COUNT_VECT(INPUT,')')) [INDEX]

(2) CLASS[ID_OPS]

(3) ASSOC[ID_OPS]

(4) POS(ID_OPS) $=1, \ldots, q$, where $q=\mid$ ID_OPS $\mid$.

We then obtain PREC(ID_OPS) $=$ POS(ID_OPS) $*$ ASSOC[ID_OPS] +2 * $(q+1) *$ CLASS[ID_OPS] $+2(q+1) *(n+2) *$ NEST(ID_OPS), where $n$ is (as usual) the number of operator classes.

PREC(ID_OPS) encodes the type, position, and parenthesis level of each identifier and operator in ID_OPS. Note that all identifiers are treated as if they were in an operator class of priority $n+1$ (i.e., higher than all other classes). This reflects the fact that all identifiers are considered to be "evaluated" before any operators are applied. It should be clear that (given $q$ and $n$ ) the encoding is unique because it can easily be uniquely inverted. The entire calculation of PREC(ID_OPS) is highly concurrent and efficient (linear in the size of INPUT).

As an example, using the same operator classes as before, let INPUT (before

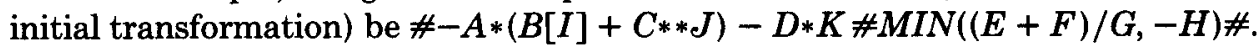
After initial transformations this becomes \#neg $A *(B$ index $(I)+C * * J)-D * K$ $\# M I N$ arg $((E+F) / G)$ apply $(\operatorname{neg} H) \#$.

In this case $q=27$ and $n=5$. PREC(ID_OPS) $=-1,114,339,164,733,666$, $1127,440,737,626,739,44,349,154,351,-16,353,262,1139,820,1141,538,751$, $256,529,754,-27$.

Given the derivation tree of an arithmetic infix expression we may define a derivation tree (called a reduced derivation tree) which involves only IDs and operators as follows:

(1) delete the leftmost and rightmost \#s and all parentheses;

(2) let $\theta$ be any binary operator, $\gamma$ any unary operator, $N$ any nonterminal, and 


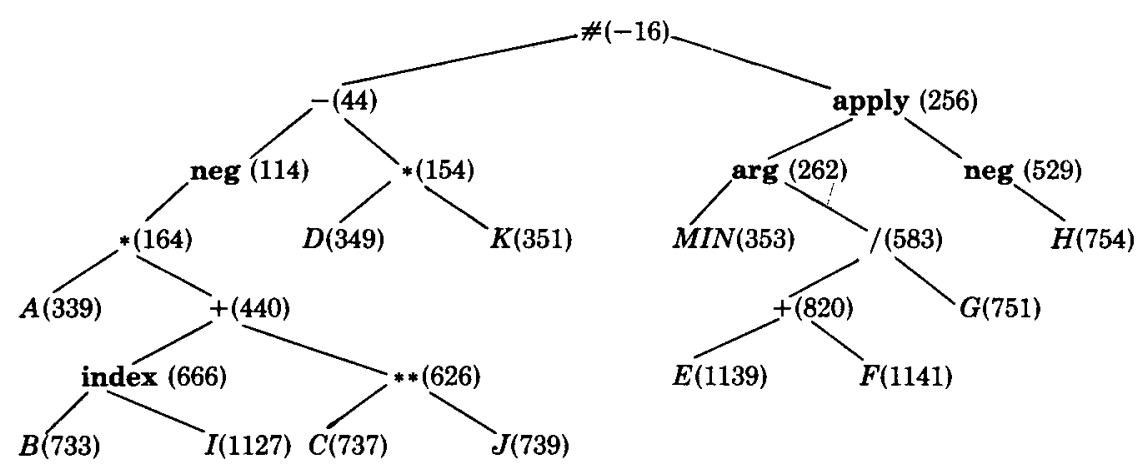

Fig. 1. Reduced derivation tree.

$T_{1}$ and $T_{2}$ arbitrary subtrees;

(i) replace $N$ with $T_{1}$;<smiles>[AlH][AlH]</smiles>

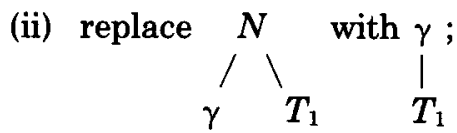

(iii) replace $N$ with $\theta$.

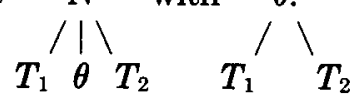

The reduced derivation tree (labeled with PREC values) corresponding to the above example is shown in Figure 1.

Observe that the PREC values have the property that all nodes (e.g., operators and identifiers) occurring in a subtree rooted by $\theta$ have a larger PREC value than that of $\theta$. This, of course, is by no means accidental.

LEMMA 4.1 Let some node $\theta_{i}$ occur in a subtree rooted by the operator $\theta_{j}$ in the reduced derivation tree of some arithmetic infix expression. Then PREC(ID_ OPS) [i] > PREC(ID_OPS) [j].

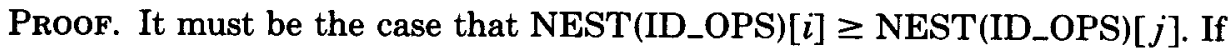
NEST(ID_OPS) $[i]=$ NEST(ID_OPS) $[j]$ then CLASS $\left[\theta_{i}\right] \geq \operatorname{CLASS}\left[\theta_{j}\right]$. If $\operatorname{CLASS}\left[\theta_{i}\right]=\operatorname{CLASS}\left[\theta_{j}\right]$, then either ASSOC[CLASS $\left.\left[\theta_{i}\right]\right]=-1$ and $i<j\left(\theta_{i}\right.$ is to the left of $\left.\theta_{j}\right)$ or ASSOC[CLASS $\left.\left[\theta_{i}\right]\right]=1$ and $i>j\left(\theta_{i}\right.$ is to the right of $\left.\theta_{j}\right)$. But these conditions imply that PREC(ID_OPS) [i] > PREC(ID_OPS) $[j]$.

The reduced derivation tree of an expression contains almost exactly the same structure as the corresponding nonreduced derivation tree (only nonterminals, endmarkers, and parentheses are lost). We will therefore consider the problem of parsing an expression to be that of determining its reduced derivation tree. The following functions are a key to such a determination.

Let $Q$ be a vector of integers. Then define

$$
\begin{aligned}
& S R(i, Q)=\{j|i<j \leq| Q \mid \text { and } \neg \exists k(i<k \leq j \text { and } Q[k]<Q[i])\} \\
& S L(i, Q)=\{j \mid 1 \leq j<i \text { and } \neg \exists k(j \leq k<i \text { and } Q[k]<Q[i])\}
\end{aligned}
$$


$S R$ scans right from $i$, getting the largest set of consecutive integers having $Q$ values greater than $Q[i]$. SL does the same scanning left. We then define

$$
\begin{aligned}
L(i, Q) \equiv & \text { if } S L(i, Q)=\varnothing \\
& \text { then } 0 \text { else } j \in S L(i, Q) \text { such that } \forall k \in S L(i, Q) Q[j] \leq Q[k] \\
R(i, Q) \equiv & \text { if } S R(i, Q)=\varnothing \\
& \text { then } 0 \text { else } j \in S R(i, Q) \text { such that } \forall k \in S R(i, Q) Q[j] \leq Q[k] .
\end{aligned}
$$

$L(R)$ simply finds an index in $S L(S R)$ that has the smallest $Q$ value, or returns 0 . Further, we define $L\left(\left(i_{1}, i_{2}, \ldots, i_{m}\right), Q\right) \equiv L\left(i_{1}, Q\right), L\left(i_{2}, Q\right), \ldots, L\left(i_{m}, Q\right) . R$ is extended in a similar manner. The import of these functions is established in the following lemma.

LEMMA 4.2 Let INPUT $\in L(G)$ where $G$ is an AIG and let PREC(ID_OPS)

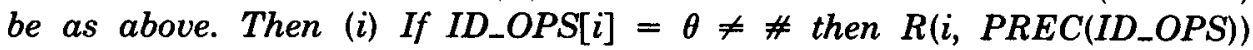
$\left(L\left(i, P R E C\left(I D \_O P S\right)\right)\right)=$ the index of the node that roots the right (left) subtree of $\theta$ if that subtree is nonnull; otherwise 0 . (ii) If ID_OPS[i] = \# is the jth \# in ID_OPS and $i<\left|I D \_O P S\right|$ then $R\left(i, P R E C\left(I D \_O P S\right)\right)=$ the root of the $j$ th expression in ID_OPS.

Proof. (i) Consider $R$ ( $L$ is analogous). All operators in $\theta$ 's right subtree are to the immediate right of $\theta$ and by Lemma 4.1 their indices will be included in $S R(i$, PREC(ID_OPS)). Let ID_OPS[ $j]=\gamma(j>i)$ be the first operator or identifier that is to the right of $\theta$ but not in $\theta$ 's right subtree. Then $\gamma$ must either be a \# (which has a PREC value smaller than any other kind of node) or an ancestor of $\theta$ (otherwise $\theta$ and $\gamma$ would have a common ancestor lying between them).

Therefore PREC(ID_OPS) $[i]>$ PREC(ID_OPS) $[j]$ and we conclude $S R(i$, PREC(ID_OPS)) is exactly the set of indices of the nodes in $\theta$ 's right subtree. But then by Lemma 4.1, $R(i$, PREC(ID_OPS)) is the root of $\theta$ 's right subtree.

(ii) By definition, if ID_OPS[i] = \# then PREC(ID_OPS) $[i]=-i$. Thus the first node to the right of this node with a smaller PREC value is the next \#. Therefore $S R(i$, PREC(ID_OPS)) is exactly the set of indices of the nodes in the $j$ th expression. The desired result then follows.

The $L$ and $R$ functions are just what is needed to determine the reduced derivation tree. With them, the roots of each individual expression as well as the immediate offspring of each operator can be readily computed. In fact, if we assume algorithms for computing $L$ and $R$ are available (this is the topic of Section 5), then a very simple AIG parser is possible.

Algorithm 4.3 (An AIG parser)

Input: One or more arithmetic infix expressions, separated by \#'s stored in INPUT.

Output: Encodings of the reduced derivation trees of the arithmetic infix expressions stored in EXPR_ROOTS, LEFT_SUBTREE, and RIGHT_SUBTREE.

1. If INPUT does not satisfy conditions (1) to (4) of the well-formedness requirement, then stop and signal a syntax error in INPUT.

2. Compute ID_OPS and PREC(ID_OPS). 
3. ('Compute positions of all but rightmost \# and positions of all non-\#'s*) IND $:=1, \ldots, \mid$ ID_OPS $\mid-1$ \#INDEX := (ID_OPS[IND] = \#) compress IND NON\#INDEX $:=($ ID_OPS $\neq \#$ ) compress $(1, \ldots, \mid$ ID_OPS $\mid)$.

4. EXPR_ROOTS $:=R$ (\#INDEX, PREC(ID_OPS)) LEF'T_SUBTREE[NON\#INDEX] $:=L($ NON\#INDEX, PREC(ID_OPS)) RIGHT_SUBTREE[NON\#INDEX] $:=R($ NON\#INDEX, PREC(ID_OPS)).

The encodings of the expressions are quite straightforward. If EXPR_ ROOTS $[i]=j$, then the root of the $i$ th expression is ID_OPS[ $j]$. In like manner, if ID_OPS $[k] \neq \#$, then LEFT_SUBTREE $[k]$ and RIGHT_SUBTREE $[k]$ yield the indices of the roots of the left and right subtrees of ID_OPS[ $k]$ or yield 0 if a subtree is null. Applying Algorithm 4.3 to our earlier example we get

EXPR_ROOTS $=12,24$

LEFT_SUBTREE $=\mathrm{NC}, 0,0,3,0,5,0,6,0,9,0,2,0,13,0, \mathrm{NC}, 0,17,0,19,0,20,0,18,0$, $0, \mathrm{NC}$

RIGHT_SUBTREE $=\mathrm{NC}, 4,0,8,0,7,0,10,0,11,0,14,0,15,0, \mathrm{NC}, 0,22,0,21,0,23,0$, $25,26,0, \mathrm{NC}$

where NC denotes not computed.

The reader may readily verify that the reduced derivation trees of the two expressions are correctly encoded. We can now establish the correctness of Algorithm 4.3 (its time and space requirements will be considered in Section 5).

Theorem 4.4 Let $G$ be some AIG. Then (i) if INPUT $\in L(G)$, then Algorithm 4.3 produces a correct encoding of the reduced derivation tree(s) of the arithmetic expression(s) in INPUT; (ii) if INPUT $\notin L(G)$, then Algorithm 4.3 will signal a syntax error and stop.

Proof. Condition (i) follows from Lemmas 3.1 and 4.2. Condition (ii) follows from Lemma 3.1.

Algorithm 4.3 is very compact and makes extensive use of the vector operations assumed available. Equally important, it is able to transform encodings of input expressions (i.e., PREC vectors) directly into encodings of the corresponding reduced derivation trees (the EXPR_ROOTS, LEFT_SUBTREE, and RIGHT_ SUBTREE vectors). The usual overhead of finding and replacing occurrences of productions is avoided.

\section{COMPUTING THE L AND $R$ FUNCTIONS}

We may now consider the problem of computing $L$ and $R$. Clearly, the two functions are symmetric so we will concentrate on computing the $R$ function. It is an easy matter to modify the algorithm to be presented to compute the $L$ function.

Let catenate be an operator which catenates together two vectors or a vector and a scalar. Further, let $a$ repeat $b$ be the vector $(b, b, \ldots)$ of length $a$.

Algorithm 5.1 (Compute $R$ function)

Input: Integer vectors $I$ and $Q$ where $1 \leq I \leq|Q|$.

Output: $R(I, Q)$.

1. ( ${ }^{*}$ Let big be any integer greater than $\left.\operatorname{MAX}(\operatorname{ABS}(\mathrm{Q}))^{*}\right)$

qsize $:=|Q|+1$

INDEX $:=1, \ldots,|I|$ 
$Q I:=((1, \ldots,|Q|)+q \operatorname{size} * Q)$ catenate $(-1 * b i g * q s i z e)$

MINVALS $:=|I|$ repeat (big*qsize)

pos $:=0$

2. loop

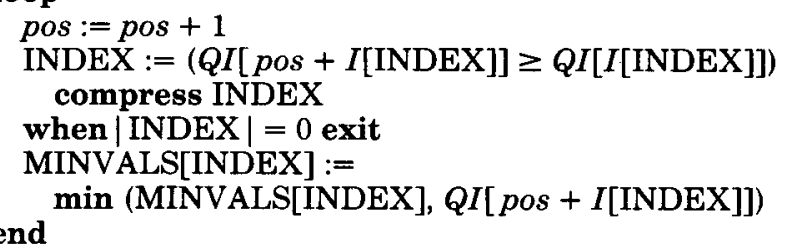

3. $R(I, Q):=$ MINVALS $\bmod$ qsize

For a given element of $I, I[j]$, Algorithm 5.1 operates by examining in turn $Q[I[j]], Q[I[j]+1]$, etc., until all of $S R(I[j], Q)$ has been examined. It is clear that $L$ could be computed by scanning left rather than right. Further, as we can now establish, the speed of Algorithm 5.1 is determined by the size of the $S R$ sets that must be examined.

THEOREM 5.2 If Algorithm 5.1 is executed on inputs $I$ and $Q$ where $|I| \leq|Q|$ then (i) $R(I, Q)$ is correctly computed; (ii) $O(|Q|)$ space is required; and (iii) $O\left(|Q|+\sum_{1 \leq j \leq|I|}|S R(I[j], Q)|\right)$ time is required.

Proof. (i) Observe that $Q I[j]$ is an encoding of $Q[j]$ and $j$ such that $Q I[k]$ $\geq Q I[l]$ if and only if $Q[k] \geq Q[l]$. Further, $Q I[j] \bmod q s i z e \equiv j$. Now consider an arbitrary component of $I, I[j] . Q I[I[j]+1], Q I[I[j]+2], \ldots$, are examined until an element $Q I[I[j]+k]$ is found such that $Q I[I[j]+k]<Q I[I[j]]$. This must occur because the last element of $Q I$ is, by construction, smaller than all others. The set of $Q I$ elements considered is exactly that corresponding to indices in $S R(I[j], Q)$. The value accumulated in MINVALS $[j]$ is therefore $\min \left(\right.$ big*qsize, $\left.\min _{l \in S R([I], Q)}(Q I[l])\right)$. If $S R(I[j], Q)=\varnothing$, then in step 3 we have $R(I, Q)[j]=($ big*qsize $) \bmod q s i z e=0$ as required; otherwise this min yields some value $Q I[m]$ for $m \in S R(I[j], Q)$. But this implies (as noted above) $Q[m]$ $=\min _{l \in S R(I[j], Q)} Q[l]$. Thus $R(I, Q)[j]=Q I[m] \bmod q s i z e=m$ as required. Therefore each element of $R(I, Q)$ is computed correctly.

(ii) Each vector used is bounded by $|Q|$.

(iii) Steps 1 and 3 clearly require at most $O(|Q|)$ time. Each iteration of step 2 requires $O(p)$ time where $p$ is the size of INDEX during that iteration. As noted in (i), a given value $j$ remains in INDEX only until all of $S R(I[j], Q)$ has been examined. This is $1+|S R(I[j], Q)|$ iterations. Thus the time needed for step 2 is $O\left(\sum_{1 \leq j \leq|I|}(1+|S R(I[j], Q)|)\right)$, and since $|I| \leq|Q|$ the desired result follows.

Algorithm 5.1 may be considered an attractive algorithm as it makes extensive use of vector operations. It is especially efficient when the number of iterations of the loop in step 2 is small, that is, when the size of the $S R$ sets is small.

We may now explore the ramifications of using Algorithm 5.1 to compute $R(I, Q)$ (and its obvious extension to compute $L(I, Q)$ ) in the AIG parser, Algorithm 4.3. Let MAX_EXPR(INPUT) be 1 if INPUT is not well formed and the size of the largest expression in ID_OPS otherwise. ${ }^{4}$ We can then establish the following.

\footnotetext{
${ }^{4}$ Recall ID_OPS is obtained from INPUT by deleting all parentheses.
} 
Theorem 5.3 Let Algorithm 4.3 use Algorithm 5.1 to compute the $R$ function and the natural extension of Algorithm 5.1 to compute the L function. If Algorithm 4.3 is executed on INPUT, then (i) it will require $O(|I N P U T|)$ space; (ii) it will require at most $O\left(|I N P U T| * M A X \_E X P R(I N P U T)\right)$ time.

Proof. (i) All vectors used are at most | INPUT | in size.

(ii) Steps 1 to 3 of Algorithm 4.3 require $O(\mid$ INPUT |) time. If INPUT is not well formed, only step 1 is executed and the result follows. Otherwise, observe that in step 4 of Algorithm 4.3 no left or right scan ever proceeds past the first \# encountered (only \#'s have negative PREC values and these values become smaller as one proceeds right). Thus the size of all $S R$ and $S L$ sets can be bounded by MAX_EXPR(INPUT) and step 4 requires (from Theorem 5.2) no more than $O(|\mathrm{INPUT}| *(1+$ MAX_EXPR(INPUT)). The desired result follows immediately.

Observe that in the worst case $\mid$ INPUT $\mid=$ MAX_EXPR(INPUT) +2 (e.g., in $\# . . .-A \#)$. Thus a quadratic execution time can result. Recall, however, that this parser is specifically designed to process large numbers of individual expressions at one time. Empirical studies [11], as well as everyday experience, indicate that individual expressions tend to be quite small. Indeed MAX_EXPR(INPUT) is not really a measure of the size of INPUT at all but rather of its complexity. For the expected class of INPUTs in which the size (and thus complexity) of individual expressions is bounded, a linear dependence of execution time on the size of INPUT can be expected.

\section{EXPRESSION TREE TRAVERSAL AND SEMANTIC PROCESSING}

Once the structure of the reduced derivation trees has been determined, it is possible to consider semantic processing and code generation questions. To perform these tasks, it is necessary to traverse derivation trees in certain specific orders. Two particular traversal orders will be considered, top-down and bottomup. During top-down traversal, the roots of the individual derivation trees are visited first. Next immediate descendants are visited, then their immediate descendants, etc., until all nodes in each derivation tree have been visited.

During bottom-up traversal, the leaves (i.e., identifiers) of the individual derivation trees are visited first. Next immediate ancestors, all of whose offspring have already been visited, are visited. This is repeated until all nodes of each derivation tree are visited.

A top-down traversal is used to convey information down derivation trees while a bottom-up traversal is used to convey information up the derivation trees. It is possible to define iterators which will perform such traversals. These iterators can then be used as high-level control structures whose bodies process nodes as they are visited. We first consider a top-down iterator.

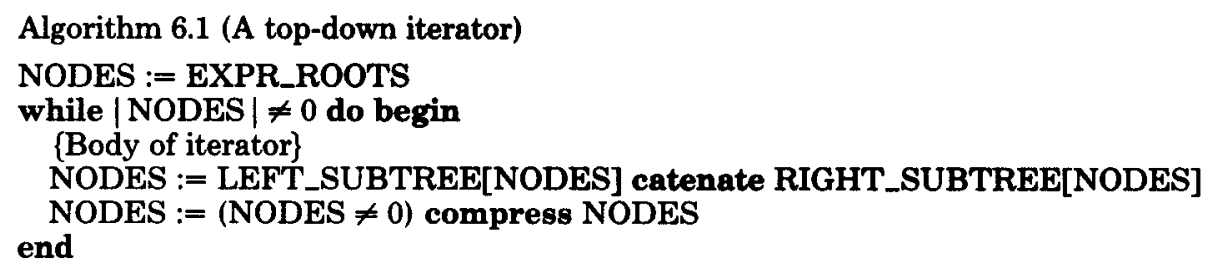


It is easy to verify that Algorithm 6.1 visits nodes in a top-down order. Further, excluding the time required to execute the iterator's body, Algorithm 6.1 requires time proportional to the number of nodes visited. Assume Algorithm 6.1 may be invoked as a control structure:

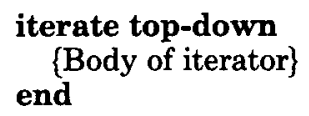

We may use Algorithm 6.1 to define a PARENT vector which, for each node, will identify its parent ( 0 will denote no parent).

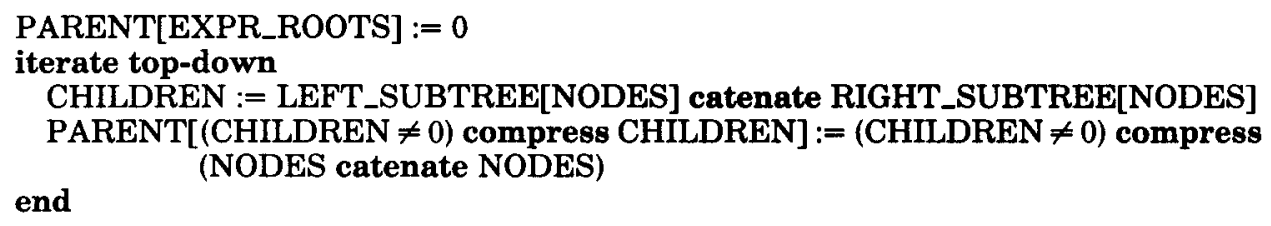

The PARENT vector can then be used in a bottom-up iterator analogous to Algorithm 6.1. Let INDEX_RANGE $(V)=i \ldots j$ be a declaration that vector $V$ is to have an index range of $i$ to $j$ (the default is $1 \ldots|V|$ ).

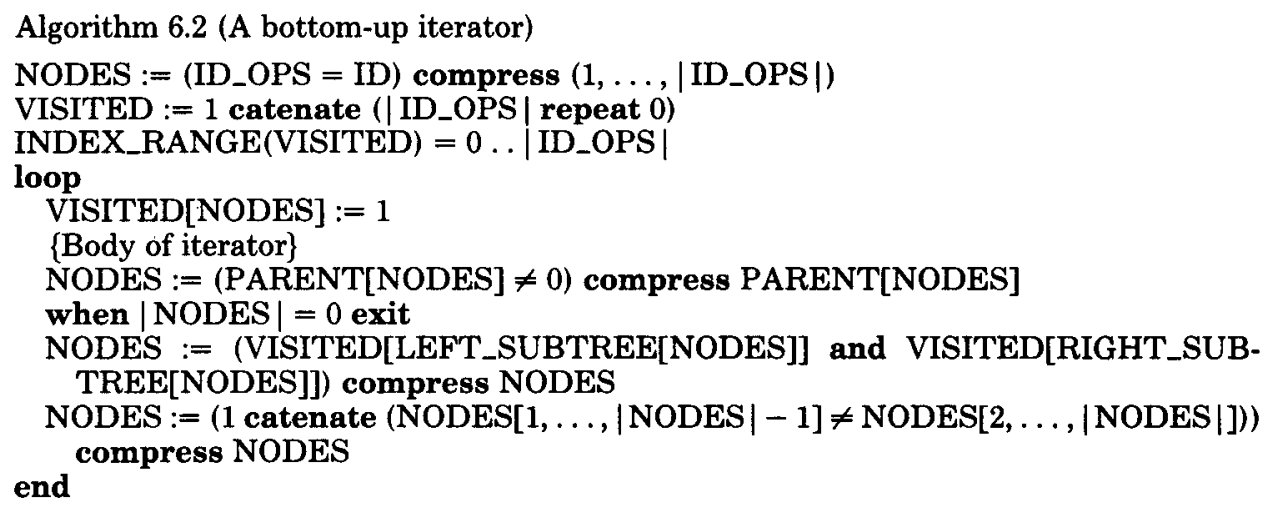

This algorithm is a bit more involved than Algorithm 6.1. In particular, it is necessary to delay visiting a node until all its children have been visited. Further, if both children of a node are visited during a given iteration, their parent will appear twice during the next iteration. It is therefore necessary to delete the second (redundant) occurrence (as is done in the last line of Algorithm 6.2). It is not difficult to verify that Algorithm 6.2 does in fact visit nodes in a bottom-up manner and further that, excluding the iterator's body, it executes in time proportional to the number of nodes visited. Like Algorithm 6.1, Algorithm 6.2 may be invoked as a control structure:

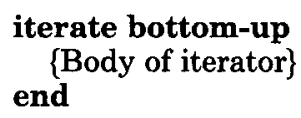

\{Body of iterator\}

A number of operations associated with semantic processing and code generation may readily be performed during a bottom-up traversal of an expression tree. These include determination of the type of an expression (or subexpression), resolution of overloaded operators (e.g., does + denote scalar or vector addition, 
Table I

\begin{tabular}{lccc}
\hline & \multicolumn{3}{c}{ Type 2 } \\
\cline { 2 - 4 } Arithmetic \\
\cline { 2 - 4 } operators & Scalar & Array & Function \\
\hline Type 1 & & & \\
Scalar & 1 & 2 & 4 \\
Array & 2 & 3 & 4 \\
Function & 4 & 4 & 4 \\
\hline
\end{tabular}

etc.), determination of code sequences required to evaluate an operator (given its operands), etc. Bottom-up traversal is well suited to these kinds of operations because the result associated with a given node is determined by that node as well as the results associated with its children (if any). Thus the type of $A+B$ depends on + , as well as the types of $A$ and $B$. In fact, a routine to determine result types of expressions (and test for invalid type usage) may be outlined as

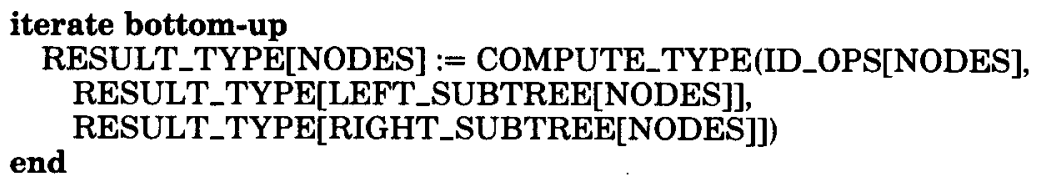

where RESULT_TYPE[0] $\equiv$ NONE (i.e., a null subtree has no type). We assume values of RESULT_TYPE are initialized for identifiers (e.g., by the scanner) before COMPUTE_TYPE is invoked.

The details of COMPUTE_TYPE will naturally depend on the various combinations of operators and types which a language allows (including possible type conversions). We can observe that various kinds of calculations will probably be needed to compute a result type and they will in general depend on the exact combination of operator and operand types involved. It is therefore useful to define classes of result type calculations. Thus all arithmetic operations involving scalars might represent one class while all array index operations might represent another.

A calculation of the following form can be used to determine the classes of calculations required. ${ }^{5}$

\section{RESULT_CLASS := T[OP_GROUP[NODES], TYPE_GROUP[TYPE1], TYPE_GROUP[TYPE2]]}

where NODES, TYPE1, and TYPE2 are the formal parameters of COMPUTE_ TYPE. We assume OP_GROUP maps operators into groups having similar type checks (e.g., arithmetic operators, indexing operators, etc.), while TYPE_GROUP does the same for types (e.g., scalars, arrays, functions, etc.). The $\mathrm{T}$ table corresponding to arithmetic operations might appear as given in Table $\mathrm{I}$.

In class 1 of Table I, the result type can be determined by a table look-up of the form RESULT[OPERATOR, TYPE1, TYPE2]. An invalid combination will yield a special "error" type. In class 2 the result is an array of the same size as the array operand. Its component type can be determined by a table look-up (as in

\footnotetext{
${ }^{5}$ Most vector machines do not support multidimensional arrays. However, a reference of the form $A[I, J, K]$, for example, can be translated to $A[V 2[V 1[I]+J]+K]$.
} 
class 1) using the operator, the scalar type, and the array component type as indices. Class 3 is similar to class 2 except that both array operands must be checked for equivalent structure. Finally in class 4 function operands are checked for free (unbound) parameters. If all parameters are bound, the function is replaced with its result type (in effect called) and the result type of the expression is then determined using the updated operand types.

Once RESULT_CLASS is computed, its values may be used to select components of the input vectors for processing. Thus operands in class 4 might first be checked and replaced with their result type. Then classes 2 and 3 might be processed to determine the array structure of their results. In these classes array types could be replaced with their component types and then operands in all four classes could be processed to determine a scalar result type. In classes 2 and 3 these scalar types would be used as the array component types.

Naturally, this approach will work best when relatively few computation classes exist. However, even when the semantics of the language being compiled require a large number of computation classes, this approach serves to effectively recognize those cases in which concurrent evaluation of result types is possible. Finally, observe that much related semantic processing can also be dealt with in a similar manner. Thus as result types were being computed, necessary type conversions, object code sequences, etc., could be determined by extending the contents of various table entries.

\section{CODE GENERATION QUESTIONS}

In Section 6 it was noted that individual operators could be labeled with the code sequences needed to evaluate the subexpressions they root. More properly, these code sequences should be considered code skeletons as they are not yet fully specified. In particular, the order in which subexpressions are to be evaluated has not yet been determined. Further, the question of how to allocate temporary storage locations (used to hold intermediate results) must be considered.

Code must always be generated so that all operands of an expression are evaluated before it is evaluated. A top-down traversal of expression trees will provide for a simple code ordering algorithm which satisfies this requirement. In many cases temporary storage locations, especially scalar or vector registers [7], are limited in number. It is therefore important to employ temporary allocation algorithms which reuse temporaries in an expression whenever possible. Two distinct allocation methods will be considered, each of which is suitable for a particular class of target machines.

To formalize these problems, we shall assume each operator in ID_OPS is to be labeled with two values, EXPR_ADR and RESULT_TEMP. EXPR_ADR will specify the address at which the code skeleton associated with the operator is to be loaded. RESULT_TEMP will index (starting at 1) the temporary to be used to hold the result computed by a given operator. Two distinct target machine models will be considered, the single execution unit (SEU) model and the multiple execution unit (MEU) model.

Under the SEU model only one operation (either scalar or vector) is in progress at any instant. Instructions, as usual, are executed in sequence. Under the MEU model (applicable to the IBM 360/91 as well as the M.I.T. "data flow" machines 
[6]) more than one operation may be in progress at the same time. The instruction stream is searched and any operation whose operands are available is performed. Operations which require results of previous instructions are held until those results become available. The MEU model assumes that an unbounded number of execution units are available. As such it represents the limiting case in which all possible independent operations in an expression are able to proceed concurrently.

We consider first the problem of ordering code skeletons (that is, determining EXPR_ADR). A simple recursive definition can be used. To order code for the expression rooted by operator $\theta$, first order the code in its left subtree, then in its right subtree, then include the code for $\theta$ itself.

Let FIRST_ADR be a vector of starting addresses for each expression, and let EXPR_SIZE be the size (in instructions) of each expression. Further, let CODE_ SIZE be the size (in instructions) of the code skeleton associated with each operator and identifier (identifiers always have a CODE_SIZE of 0 ). Similarly, let RIGHT_SUBTREE_SIZE be the size (in instructions) of the entire right subtree of an operator. EXPR_SIZE, CODE_SIZE, and RIGHT_SUBTREE_ SIZE can readily be computed by a bottom-up traversal as outlined in Section 6 . FIRST_ADR is assumed to be supplied to the compiler with the expressions to be processed. The following algorithm can be used to assign instruction addresses (via EXPR_ADR).

Algorithm 7.1 (An instruction address generator)

Input: (1) Encodings of reduced derivation trees as produced by Algorithm 4.3.

(2) FIRST_ADR, EXPR_SIZE, CODE_SIZE, RIGHT_SUBTREE_SIZE.

Output: EXPR_ADR.

1. first_iter $:=$ true

2. iterate top-down

if first_iter then begin

first_iter := false

EXPR_ADR[NODES] :=FIRST_ADR + EXPR_SIZE-CODE_SIZE[NODES] end

OPS $:=($ KIND[NODES] $\neq$ IDENTIFIER) compress NODES

EXPR_ADR[RIGHT_SUBTREE[OPS]] :=

EXPR_ADR[OPS]-CODE_SIZE[RIGHT_SUBTREE[OPS]]

LEFT_CHILDREN $:=$ LEFT_SUBTREE[OPS] $\neq 0$

EXPR_ADR[LEFT_CHILDREN compress LEFT_SUBTREE[OPS] := EXPR_ADR[LEFT_CHILDREN compress OPS]-

RIGHT_SUBTREE_SIZE[LEFT_CHILDREN compress OPS]end

CODE_SIZE[LEFT_CHILDREN compress LEFT_SUBTREE[OPS]]

The algorithm first assigns addresses to the expression roots. These follow all other instructions. It then proceeds down the expression trees.

A right subtree is assigned an address immediately preceding its parent and a left subtree is assigned an address preceding its corresponding right subtree.

A very simple temporary allocation scheme may be used with the SEU model. Each operator is labeled with an integer $i$. The operator must store its result into $T_{i}$ (the $i$ th temporary). Further, any $T_{j}(j \geq i)$ may be used in evaluating the operator's subtrees. The root of each expression is labeled with 1 . Given the label of an operator, labels for left and right children are determined as follows: If an 
operator has label $i$, then its left child (if it is an operator) also has label $i$. If the right child is an operator then if the left child is an operator, the right child is labeled with $i+1$; otherwise it is labeled with $i$. This leads to the following algorithm which computes values of RESULT_TEMP.

Algorithm 7.2 (An SEU temporary allocator)

Input: Encodings of reduced derivation trees as produced by Algorithm 4.3. Output: RESULT_TEMP.

1. first_iter $:=$ true

2. iterate top-down OPS $:=($ KIND $[$ NODES] $\neq$ IDENTIFIER) compress NODES

if first_iter then begin

first_iter := false

RESULT_TEMP[OPS] := 1 end

RESULT_TEMP[(LEFT_SUBTREE[OPS] = 0) compress RIGHT_SUBTREE[OPS] :=

RESULT_TEMP[(LEFT_SUBTREE[OPS] = 0) compress OPS]

OPS $:=($ LEFT_SUBTREE[OPS] $\neq 0$ ) compress OPS

LEFT_IDS := KIND[LEFT_SUBTREE[OPS]] = IDENTIFIER

RESULT_TEMP[(not LEFT_IDS) compress LEFT_SUBTREE[OPS] := RESULT_TEMP[(not LEFT_IDS) compress OPS]

RESULT_TEMP[LEFT_IDS compress RIGHT_SUBTREE[OPS]] := RESULT_TEMP[LEFT_IDS compress OPS]

RESULT_TEMP[(not LEFT_IDS) compress RIGHT_SUBTREE[OPS] := end $1+$ RESULT_TEMP[(not LEFT_IDS) compress OPS]

Note that in some cases a value of RESULT_TEMP will be stored for identifiers. As these values are not used, they may be ignored. We may illustrate Algorithms 7.1 and 7.2 using the example of Section 4. Assume (for simplicity) that all code skeletons are of unit size and that instructions may be represented as quadruples of the form (OP, $A, B, T$ ), where OP is an operator, $A$ and $B$ are either temporaries or identifiers, and a quadruple is assumed to have the semantics $T:=A$ OP $B$ ( $A$ is null for unary operators). The code generated for the two expressions in the earlier example is illustrated below (ordered by EXPR_ADR):

$$
\begin{aligned}
& \text { (index, } B, I, T_{1} \text { ) } \\
& \text { (**, } \left.C, J, T_{2}\right) \\
& \text { (+, } \left.T_{1}, T_{2}, T_{1}\right) \\
& \text { (*, } \left.A, T_{1}, T_{1}\right) \\
& \text { (neg, } \left.T_{1}, T_{1}\right) \\
& \text { (*, } \left.D, K, T_{2}\right) \\
& \text { (- } \left., T_{1}, T_{2}, T_{1}\right)
\end{aligned}
$$

$$
\begin{aligned}
& \left(+, E, F, T_{1}\right) \\
& \left(/, T_{1}, G, T_{1}\right) \\
& \left(\arg , M I N, T_{1}, T_{1}\right) \\
& \left(\text { neg, } H, T_{2}\right) \\
& \text { (apply, } \left.T_{1}, T_{2}, T_{1}\right)
\end{aligned}
$$

It is easy to verify that the code sequences are correct. The following establishes the properties of the above algorithms.

Theorem 7.3 Assume Algorithm 4.3 is executed on INPUT, a vector of syntactically and semantically correct arithmetic infix expressions. Assume further that the reduced derivation trees are correctly labeled with code skeletons and Algorithms 7.1 and 7.2 are executed using these derivation trees. Then

(i) the code sequences generated will correctly evaluate the arithmetic infix expressions specified by the reduced derivation trees on an SEU computer; 
(ii) given the evaluation order specified by Algorithm 7.1, Algorithm 7.2 will allocate the fewest possible temporaries for each expression;

(iii) Algorithms 7.1 and 7.2 will execute in at most $O(|I N P U T|)$ time and space.

Proof. (i) Consider an induction on $h$, the height of derivation trees (or subtrees) for which code is generated. For $h=1$, all operands are IDs and correct code follows from correct code skeletons. Next consider a tree $T$ of height $h+1$. All of $T$ 's subtrees are of height $h$ or less and the induction hypothesis holds for them. If $T$ has only one subtree that is not an ID, code to evaluate that subtree immediately precedes code to evaluate $T$ 's root and the result follows. Otherwise, code to evaluate $T$ 's left subtree is immediately followed by code to evaluate $T$ 's right subtree which is immediately followed by code to evaluate $T$ 's root. If RESULT_TEMP for $T$ 's root is $i$, then the left subtree is evaluated (correctly) into $T_{i}$ and the right subtree is evaluated (correctly) into $T_{i+1}$. By construction, the right subtree cannot use any temporary with index less than $i+1$, so when code for $T$ 's root is executed, both $T_{i}$ and $T_{i+1}$ must contain correct operand values and the result follows.

(ii) We must show that the RESULT_TEMP value for each operator is the smallest possible; that is, that all lower numbered temporaries are in use. This clearly holds for the root of each expression which has a RESULT_TEMP value of 1. Assume the root of a tree (or subtree) has a RESULT_TEMP value of $i$ which is the smallest possible. Consider its immediate offspring. If only one is a non-ID, it has a RESULT_TEMP value of $i$ which is clearly minimal. Otherwise it has a left child with a RESULT_TEMP value of $i$ and a right child with a RESULT_TEMP value of $i+1$. The left child's value is clearly minimal. Since the left child is evaluated first, its result is held in $T_{i}$, so the right subtree cannot have a RESULT_TEMP value less than $i+1$.

(iii) Observe that $\mid$ ID_OPS $|\leq|$ INPUT $\mid$. In both algorithms, the time and space required for one top-down iteration is proportional to |NODES|. Since each node in ID_OPS appears in NODES only once, the result follows.

If an execution order other than that specified by Algorithm 7.1 is used, it is sometimes possible to use fewer temporaries. This optimization has been studied by Sethi and Ullman [19], but is beyond the scope of this paper.

We now consider the MEU case. Algorithm 7.1 can still be used to generate instruction addresses. This is because MEU computers are able to scan down instruction sequences and evaluate those instructions whose operands are available while delaying those which depend upon operations which are not yet completed. However, Algorithm 7.2 can no longer be used to allocate temporaries. This is because this algorithm allows the same temporary to be used in two distinct subtrees. The MEU model allows two distinct subtrees to be evaluated concurrently, and if they should use the same temporary, either intermediate results needed by one subtree could be destroyed by the other or the concurrent evaluation of the two subtrees would be inhibited.

The new temporary allocation routine will allocate a distinct temporary to each operator having only IDs as operands (so that all of these may execute concurrently). If an operator has a non-ID as an offspring, it will inherit the temporary used by that offspring (arbitrarily choosing the left child if both are non-IDs). 
This ensures that only operators which are direct descendants (or ancestors) of each other can share temporaries, thus maximizing potential concurrency.

Assume that each element in ID_OPS has associated with it an element of the vector EXPR\#, denoting the expression in which the node appears (starting at 1). This can easily be determined by a top-down traversal of the expression trees. ${ }^{6}$ The following algorithm can be used to allocate temporaries for an MEU machine.

\section{Algorithm 7.4 (An MEU temporary allocator)}

Input: (1) Encodings of reduced derivation trees as produced by Algorithm 4.3.

(2) The vector EXPR\#.

Output: RESULT_TEMP.

1. iter_num :=0

2. iterate bottom-up

iter_num := iter_num +1

if iter_num $=2$ then begin

LEFT_EXPR_POS $:=1$ catenate

$(($ EXPR\# $[$ NODES $[1, \ldots, \mid$ NODES $\mid]] \neq$

EXPR\# [NODES $[2, \ldots, \mid$ NODES $\mid]])$ compress $(2, \ldots, \mid$ NODES $\mid))$

RESULT_TEMP[NODES] :=

$1+(1, \ldots, \mid$ NODES $\mid)-L E F T \_E X P R \_P O S[E X P R \#$ [NODES]]

end else if iter_num $>2$ then begin

RESULT_TEMP[(LEFT_SUBTREE[NODES] $=0$; compress NODES] :=

RESULT_TEMP[(LEFT_SUBTREE[NODES] = 0) compress RIGHT_SUB-

TREE[NODES]]

$\mathrm{N}:=($ LEFT_SUBTREE[NODES] $\neq 0$ ) compress NODES

LEFT_IDS $:=$ KIND[LEFT_SUBTREE[N]] = IDENTIFIER

RESULT_TEMP[LEFT_IDS compress $N$ ] :=

RESULT_TEMP[LEFT_IDS compress RIGHT SUBTREE[N]]

RESULT_TEMP[(not LEFT_IDS) compress $\mathrm{N}]:=$ end

RESULT_TEMP[(not LEFT_IDS) compress LEFT_SUBTREE[N]]

end

The first iteration skips past all identifiers. During the second iteration, the leftmost operator of each expression in NODES is found. These operators are assigned a RESULT_TEMP of 1. Other operators are assigned values according to their positions relative to the leftmost operators (i.e., if expression $e$ 's leftmost operator is at position $i$, then an operator in expression $e$ at position $j$ gets a RESULT_TEMP value of $1+j-i$ ). On succeeding iterations RESULT_TEMP values are inherited from a left or right child.

If we reconsider our earlier example, this time using Algorithm 7.4 to allocate temporaries, we find that the code for the second expression is unchanged but that generated for the first expression is a bit different:

(1) (index, $B, I, T_{1}$ )

(2) $\left(* *, C, J, T_{2}\right)$

(3) $\left(+, T_{1}, T_{2}, T_{1}\right)$

(4) (*, $\left.A, T_{1}, T_{1}\right)$

(5) (neg, , $T_{1}, T_{1}$ )

(6) $\left(*, D, K, T_{3}\right)$

(7) $\left(-, T_{1}, T_{3}, T_{1}\right)$

\footnotetext{
${ }^{6}$ Algorithm 3.2 can also be used to compute EXPR\# by "counting" the \# symbols in the original input.
} 
A third temporary has been used in quadruple (6), indicating that three concurrent operations are possible (quadruples (1), (2), and (6)).

A result analogous to Theorem 7.3 can be established.

Theorem 7.5. Assume Algorithm 4.3 is executed on INPUT, a vector of syntactically and semantically correct arithmetic infix expressions. Assume further that the reduced derivation trees are correctly labeled with code skeletons and that Algorithms 7.1 and 7.4 are executed using these derivation trees. Then

(i) the code sequences generated will correctly evaluate the arithmetic infix expressions specified by the reduced derivation trees on an MEU computer;

(ii) given an MEU computer with an unbounded number of execution units, Algorithm 7.4 will allocate the fewest possible temporaries for each expression which allow all independent computations to proceed concurrently;

(iii) Algorithms 7.1 and 7.4 will execute in at most O(|INPUT|) time and space.

Proof. (i) As noted previously, Algorithm 7.1 always orders code for the left and right subtrees of an operator before code for the operator itself. Further, it is easy to verify that Algorithm 7.4 allocates temporaries so that two independent subtrees of an expression never use the same temporary. These facts, with the assumption that code skeletons are correct, yield the desired result.

(ii) For each expression, Algorithm 7.4 allocates a number of temporaries equal to the number of operators having only IDs as operands. Since these operations are clearly independent, the number of temporaries allocated is a lower bound on the minimum number needed to allow all possible concurrent operations to proceed. But two operations are able to proceed concurrently if and only if they represent independent subtrees. By construction, independent subtrees never share a temporary so all possible concurrent operations may proceed without additional temporaries.

(iii) Identical to proof of Theorem 4.3 (iii).

Optimization techniques are known which increase potential concurrency in expression evaluation by restructuring derivation trees ([5], [13], [16]). Such techniques could profitably be included in a parallel compiler for a parallel target machine.

In real MEU machines the number of available execution units is of course bounded. For such machines the code sequences produced by Algorithm 7.1 can sometimes be improved by putting independent operations before those which need earlier results (this reduces the number of instructions which must be "held" for later execution). Further, a hybrid of Algorithms 7.2 and 7.4 could be used on these machines. It would allocate enough temporaries to allow all available execution units to operate concurrently, but would reuse temporaries in those cases where the number of independent operations exceeded the number of available execution units.

Similar modifications would be of value in generating code for such machines as the CDC 7600, CDC Star, and Cray-1. Such machines do not fully meet MEU machine requirements, since once an instruction whose operands are not ready is encountered, no further instructions are issued until that instruction can proceed. Thus while the code produced by Algorithms 7.1 and 7.4 is still correct, it is not 
necessarily optimal (in terms of execution speed). Unfortunately, generation of optimal code for such machines is very difficult (even using "ordinary" compilers) as the exact number, kind, and operational details of execution units can influence code generation. Nevertheless, methods of improving generated code are very important and deserve further study.

\section{CONCLUSION}

The algorithms and techniques presented above appear to be a very promising first step toward the development of compilers which run efficiently and effectively on vector computers. As has been demonstrated, they make extensive use of vector operations, are quite compact in structure, and are linear in cases of interest. The question of how effective these algorithms and techniques would be on an actual vector machine is as yet unanswered. In large measure this is due to the fact that such machines are not yet commonly available to researchers. Certainly careful implementations and tests are called for.

At the same time research into writing entire vector compilers should be pursued. In many cases techniques used in compiling arithmetic infix expressions can be extended; in other cases, further work is needed. The problem of performing target code optimizations on vector machines deserves special attention. Certainly any compiler that is to see wide use must be able to generate quality code. Also, many known optimizations are quite expensive to perform, especially for large programs. The possibility of exploiting the high performance available on vector machines in doing such optimizations must not be overlooked.

\section{ACKNOWLEDGMENTS}

I am indebted to the referees for many thoughtful and helpful comments on earlier drafts of this paper. I am also grateful to Frank Horn for aid in editing this paper. Ben Wegbreit and Susan Graham, as Editors, were very helpful.

\section{REFERENCES}

1. A description of the advanced scientific computer system. Texas Instruments Inc., Austin, Tex., April 1973.

2. Aho, A., And Ullman, J. The Theory of Parsing, Translation, and Compiling, vol. 1. PrenticeHall, Englewood Cliffs, N.J., 1972.

3. BAER, J., AND ElLIS, C. Model, design, and evaluation of a compiler for a parallel processing environment. IEEE Trans. Softw. Eng. SE-3, 6 (Nov. 1977), 394-405.

4. Baskett, F., AND Keller, T. An evaluation of the Cray-1 computer. Presented at the Symp. High Speed Computer and Algorithm Organization, Champaign, Ill., April 13-15, 1977.

5. BRENT, R. The parallel evaluation of general arithmetic expressions. J. ACM 21, 2 (April 1974), 201-206.

6. Dennis, J., AND Misunas, D. A preliminary architecture for a basic data-flow processor. In Proc. 2nd Annu. Symp. Computer Architecture, Jan. 1975, pp. 126-132.

7. Description of Cray-1. Cray Research Corp., Chippewa Falls, Wis. 1975.

8. Donegan, M., AND KatzKe, S. Lexical analysis and parsing techniques for a vector machine. In Proc. Conf. Programming Languages and Compilers for Parallel and Vector Machines, SIGPLAN Notices 10, 3 (March 1975), 138-145.

9. Fischer, C. On parsing context free languages in parallel environments. Ph.D. dissertation, Comput. Sci. Dep., Cornell Univ., Ithaca, N. Y., 1975.

10. Hopcroft, J., AND Ullman, J. Formal Languages and Their Relation to Automata. AddisonWesley, Reading, Mass., 1969. 
11. KNUTH, D. An empirical study of FORTRAN programs. Softw. Pract. Exper. 1 (1971), $105-133$.

12. KROHN, H. A parallel approach to code generation for FORTRAN-like compilers. In Proc. Conf. Programming Languages and Compilers for Parallel and Vector Machines, SIGPLAN Notices 10, 3 (March 1975), 146-149.

13. KuCK, D., AND MaruYama, K. Time bounds on the parallel evaluation of arithmetic expressions. SIAM J. Comput. 4, 2 (June 1975), 147-162.

14. Lincoln, N. Parallel programming techniques for compilers. SIGPLAN Notices 5,10 (Nov. 1970).

15. Mickunas, M., and Schell, R. Parallel compilation in a multiprocessor environment. In Proc. ACM 1978 Annu. Conf., Dec. 1978, pp. 241-246.

16. Muller, D., and Preparata, F. Restructuring of arithmetic expressions for parallel evaluation. J. ACM 23, 3 (July 1976), 534-543.

17. Ramamoorthy, C., and Li, H. Pipeline architecture. Comput. Surv. 9, 1 (March 1977), 61-102.

18. Richards, M. BCPL: A tool for compiler writing and systems programming. Proc. AFIPS 1969 SJCC, vol. 34, AFIPS Press, Arlington, Va., pp. 557-566.

19. Sethi, R., ANd Ullman, J. The generation of optimal code for arithmetic expressions. J. ACM 17, 4 (Oct. 1970), 715-728.

20. STAR-100 Computer System. Publ. 6025600, Control Data Corp., Arden Hills, Minn., 1973.

21. Zosel, M. A parallel approach to compilation. In Proc. ACM Symp. Principles of Programming Languages, Boston, Mass., Oct. 1978; pp. 59-70.

Received April 1976; revised September 1977 and December 1979; accepted January 1980 